\title{
Nonselective motor-level changes associated with selective response inhibition: evidence from response force measurements
}

\author{
Yao-Ting Ko • Jeff Miller
}

Published online: 9 April 2011

(C) Psychonomic Society, Inc. 2011

\begin{abstract}
In the present study, we examined the effects of selective response inhibition on motor production using response force measures within a task that was based on that of Aron and Verbruggen (Psychological Science, 19, 1146-1153, 2008). In each trial, participants were signaled to respond bimanually with the two index fingers or the two middle fingers. After a short delay, a stop signal was sometimes presented, indicating that one of the two finger responses should be withheld. A given response was slowed when the response on the other hand was stopped, replicating a previously observed stopping interference effect. In addition, the given response was also made more forcefully when the response on the other hand was stopped, indicating that the requirement to stop one activated response has global motor-level consequences for other responses that are to be carried out normally.
\end{abstract}

Keywords Inhibition $\cdot$ Motor control

We can inhibit response tendencies in response to changes in our original goals. Our ability to control or redirect our thought or behavior using response inhibition is one manifestation of our executive control system.

The stop-signal paradigm has been a prominent tool for studying inhibitory acts of control arising as a result of external stimuli (Logan \& Cowan, 1984). In this paradigm, a primary-task stimulus is presented to establish an original goal, and a subsequent stop signal is occasionally presented

Y.-T. Ko $(\bowtie) \cdot$ J. Miller $(\bowtie)$

Department of Psychology, University of Otago,

Dunedin, New Zealand

e-mail: koya2759@student.otago.ac.nz

e-mail: miller@psy.otago.ac.nz to cancel or change that goal. Performance is modeled as a race between a primary-task process and a stopping process, with the primary response canceled when the stopping process finishes first.

An important feature of our ability to inhibit responses is that we can selectively inhibit a subset of several concurrently active response tendencies. This capacity for selective response inhibition is essential because we usually have several concurrent response tendencies and, often, only a subset must be stopped. Classical stop-signal tasks have not addressed selective response inhibition (Verbruggen \& Logan, 2008), however, because their primary-task stimuli activate only one response tendency, which is carried out or stopped.

Several different selective stopping tasks have been studied, but not all were selective in the sense of requiring inhibition of a subset of several concurrently active responses. For example, De Jong, Coles, and Logan (1995) had participants perform a primary task requiring a response with the left or right hand. In the selective stopping condition, the stop signal was relevant for only one prespecified response. For example, participants might have to stop left-hand responses whenever the stop signal is presented, carrying out right-hand responses normally. As was emphasized by Coxon, Stinear, and Byblow (2007), this task requires discrimination between stimuli whose responses are or are not subject to stopping. It does not address, however, the processes involved in selectively stopping one of several concurrent response tendencies, because the primary task requires only one response.

To study selective inhibition within a subset of concurrent response tendencies, Coxon et al. (2007) designed a rather different task. At the beginning of each trial, the participant held down two response keys, causing two corresponding lines to ascend toward a fixed target at the top of the display. The participant was required to release 
the two keys when the lines reached the target, so that two response tendencies were active. In the selective-stop condition, one line stopped before reaching the target, and the participant was required not to release the key corresponding to this line. The other key, however, still had to be released when its line reached the target. In the stop-all condition, both lines stopped before reaching the target, and the participant was required not to release either key. Coxon et al. found that it was harder to selectively release only one key than to nonselectively release neither key, as was indexed by the probability of successfully withholding the key(s) signaled for stopping. More importantly, comparing selective-stop and nonstop trials (i.e., no lines stopped before reaching the target), key release by one hand was delayed when the other hand's release had to be stopped. Thus, the results demonstrate an RT cost when selective stopping is required, implying that the stopping process acted nonselectively or globally to some extent.

Aron and Verbruggen (2008) used the term stoppinginterference effects (SIEs) to refer to reaction time (RT) costs associated with stopping one of several concurrently active response tendencies. Specifically, an SIE is a slowdown of the unstopped or "nontarget" response when stopping is required for the other or "target" response, relative to the nostopping condition, as was observed by Coxon et al. (2007). Aron and Verbruggen attributed this effect to a "nonselective" or "global" stopping mechanism that "first stopped both responses...and then reinitiated" the unstopped one (p. 1146). Aron and Verbruggen aimed to distinguish experimentally between this hypothesized global, rapid stopping mechanism, and another stopping mechanism that acts selectively and slowly. They assumed that participants would employ the selective mechanism when they knew in advance which hand might need to be stopped, and that the selective mechanism would produce a smaller SIE. In their experiment, an initial cue either indicated which hands might be stopped in a trial or was uninformative. Then, left- and righthand figures were presented to signal whether two simultaneous index versus middle finger bimanual responses were required. Occasionally, an X later appeared over one hand's figure, signaling that this hand should be stopped. As was predicted, the SIE was smaller with informative than with uninformative cues, so Aron and Verbruggen concluded that selective response inhibition was possible when participants knew which response might be stopped. However, it is noteworthy that the SIE was still large in the cued condition (about $100 \mathrm{~ms}$ ), suggesting that a nonselective mechanism contributes to stopping even after an informative cue.

The SIEs observed so far indicate that some process(es) involved in inhibiting the target response also delay the nontarget response. From RT measures alone, however, it is difficult to be sure in which stage(s) the nontarget response is delayed. One possibility is that the effect arises in motor processing. In nonselective stop-signal tasks in which only one response tendency is activated and subsequently inhibited, psychophysiological measures indicate that inhibition exerts its effects at and after responses are activated in the primary motor cortex (M1; e.g., De Jong et al., 1995; De Jong, Coles, Logan, \& Gratton, 1990). Such inhibitory effects on relatively late motor processing are often termed peripheral (e.g., Band \& van Boxtel, 1999). In selectivestopping tasks, it is certainly possible that analogous peripheral motor inhibition is responsible for the SIE.

There are other possibilities, however, because RT measures the time needed for all processes required for responding, not just peripheral motor processes. When the stop signal indicates which response must be stopped, for example, it might cause decision-level delays associated with selecting the target response, much like the central delays in the psychological refractory period paradigm (e.g., Pashler, 1994). In that case, the SIE would be produced centrally, with nontarget responses delayed before M1. Decision-level delays could also be responsible for the SIE when the stop signal conveys no information about which response must be stopped, as in the informative cue condition of Aron and Verbruggen (2008), because the stop signal still conveys the information that one response must be stopped rather than carried out.

Although they focused on behavioral measures, Coxon et al. (2007) presented some EMG data suggesting a motorlevel contribution to stopping interference. Specifically, they found a greater peak rate of change in nontarget-hand EMG activity in trials with successful stopping than in trials without a stop signal. This result is not decisive evidence of an SIE at the motor level, however. Most critically, Coxon et al. measured the peak rate of change in EMG waveforms averaged across trials. The shape of an average waveform is "smeared" by timing variability or "latency jitter" (e.g., Callaway, Halliday, Naylor, \& Thouvenin, 1984). This smearing contaminates comparisons between conditions with different amounts of latency jitter, as would be expected for this comparison under the standard race model of the stopsignal task (Logan \& Cowan, 1984). To avoid smearing, it is better to measure peripheral motor processing in each trialfor example, using response force. Furthermore, Coxon et al.'s design had several unusual features that made it difficult to compare with standard stop-signal tasks. For example, participants produced movement(s) at predetermined target times (i.e., when the moving lines reached the target) rather than producing the movements as rapidly as possible after the primary task stimulus. Therefore, their task allowed responses to be prepared over a relatively long time interval, potentially allowing greater coupling and more difficulty in selective inhibition.

In the present study, we tested for peripheral response inhibition in a selective stopping task using response force as 
an index of peripheral motor processing. Response force is a sensitive measure of motor processing that often provides information unavailable in RT (e.g., Giray \& Ulrich, 1993). Evidence has indicated that lower level movement parameters, such as response force or direction, are specified at M1 (see Ashe, 1997, for a review). Accordingly, measuring response force in a selective inhibition task can provide information about whether the SIE has consequences for peripheral motor processes, just as force has been used to study the locus of various other experimental effects (e.g., Giray \& Ulrich, 1993). Specifically, we asked whether selective stopping affects the forcefulness of nontarget hand responses as well as their latency (i.e., SIE on force). Forcebased SIEs would indicate that selective response inhibition leads to changes in processing at the peripheral motor level, thereby providing further support for the notion that peripheral processes are involved in response inhibition and in constraining the locus of SIEs.

\section{Method}

\section{Participants}

Participants were 20 University of Otago students, 10 women, ranging in age from 18 to 36 years $(M=23.9$ years). All had normal or corrected-to-normal vision. Each participated in a single session lasting approximately 50 min with a payment of NZD 15.

\section{Apparatus}

An IBM-compatible computer was used to present stimuli and record responses. Responses were made on four forcesensitive keys similar to telegraph keys $(140 \times 20 \times 2 \mathrm{~mm})$. Participants responded by pressing down the nearer ends using quick finger flexions. Strain gauges converted force to an analog signal with a resolution of approximately $2.8 \mathrm{mN}$. The signal from each key was digitized for $2.2 \mathrm{~s}$ at $250 \mathrm{~Hz}$, starting $200 \mathrm{~ms}$ before stimulus onset. A force criterion of $100 \mathrm{cN}$ was used in deciding whether a response had been generated for the purpose of providing accuracy feedback.

\section{Stimuli}

Four outline squares of approximately $1 \mathrm{~cm}$ per side were deployed along the central horizontal line of the screen that participants viewed from a distance of $65 \mathrm{~cm}$. Adjacent squares were separated by $0.8 \mathrm{~cm}$; two were deployed on the left of the vertical midline, and two were on the right of it. The two squares nearer the midline will be referred to as the inner squares; the other two, as the outer squares.
In each trial, either the two inner or the two outer squares were simultaneously filled in to serve as the primary-task stimulus, and these two possibilities were equally likely. A red "X" on the diagonals of one filled-in square was occasionally presented and served as the stop signal. In $40 \%$ of all trials, the stop signal was presented on one of the filled-in squares with equal probabilities for both squares.

\section{Procedure}

A single session included six blocks of 100 trials, with rest breaks between blocks. In each trial, participants were first presented with the four outline squares. After $1,000 \mathrm{~ms}$, the two inner or outer squares filled in. If the two inner squares filled in, participants were instructed to simultaneously and quickly press the keys with their index fingers; if the two outer squares filled in, they were required to simultaneously and quickly press the keys with their middle fingers.

Occasionally, shortly after the two squares filled in, the stop signal was presented, superimposed on one of the squares. Participants were instructed to stop their left-hand response while still quickly executing their right-hand response when the left stop signal was presented (i.e., red $\mathrm{X}$ on the left square). Symmetrically, they were to stop the right-hand response but to execute the left-hand response when the $\mathrm{X}$ was shown on the right. The delay from primary-task stimulus onset to stop-signal onset (i.e., the stop-signal delay or SSD) started at $100 \mathrm{~ms}$ and was dynamically adjusted for each finger. For a given finger, it increased $50 \mathrm{~ms}$ (to make stopping harder) after each successful stopping and decreased $50 \mathrm{~ms}$ (to make stopping easier) after each unsuccessful stopping. ${ }^{1}$

Participants were instructed to make their responses immediately when they saw the squares. They were also informed that it was normal that they would often be unable to stop when the red " $\mathrm{X}$ " appeared. In addition, they were told that feedback would be given to help them follow the instructions. Specifically, feedback was given after responses with the wrong finger(s), responses made despite a stop signal, responses that were too slow (RT $>600 \mathrm{~ms}$ ), and bimanual responses that were not simultaneous (IRI $>50 \mathrm{~ms}$ ).

\section{Results}

Trials with incorrect primary-task responses (i.e., index fingers instead of middle or vice versa) were rejected $(3.86 \%$ of all trials). A total of $1.75 \%$ of correct trials were

\footnotetext{
${ }^{1}$ Although the SSD could be adjusted to values smaller than zero, the computer program actually produced an SSD of at least $10 \mathrm{~ms}$.
} 
excluded on the basis of additional criteria, ${ }^{2}$ resulting in $94.46 \%$ of all trials included in further analysis and statistical testing.

The probability of successful stopping given the stop signal was 0.53 , indicating that the SSD-adjustment tracking algorithm worked well (Logan, Schachar, \& Tannock, 1997). The mean SSD was $223 \mathrm{~ms}$, and the estimated SSRT was $221 \mathrm{~ms}$, as calculated by subtracting the mean SSD from the mean RT of the no-stop signal trials. This estimated SSRT is a typical value for a normal population (e.g., Logan et al., 1997).

Three trial types were examined: No stopping was required (no-stopping trials), stopping one hand was required and successful (successful-stopping trials), and stopping one hand was required but unsuccessful (unsuccessful-stopping trials). Three dependent variables were computed in each trial. First, RTs (in milliseconds) were measured as the times needed to generate $100 \mathrm{cN}$ of force. Second, the maximum or peak value of response force $(\mathrm{PF}$, in $\mathrm{cN})$ was obtained. Third, the rate of force development (RFD, in $\mathrm{cN} / \mathrm{ms}$ ) was computed as the force difference between the criterion and the PF (i.e., PF$100 \mathrm{cN}$ ) divided by the time between crossing the criterion and reaching the PF.

\section{Simultaneous responding of the two hands}

Two lines of evidence indicated that participants activated the two responses as a single unit (i.e., grouped), as instructed. First, the mean absolute RT difference between the two hands, computed trial by trial, was $14 \mathrm{~ms}$ in no-stopping trials and $24 \mathrm{~ms}$ in unsuccessful-stopping trials. ${ }^{3}$ Second, on average, across participants, the correlation between the RTs of the two hands was 0.938 in no-stopping trials and 0.832 in unsuccessful-stopping trials, correlating across trials separately for each participant ( $p<0.01$ for each participant).

\section{Stopping effects}

Effects of stopping on target-hand performance were examined by comparing a given hand's response across no-stopping trials, successful-stopping trials, and unsuccessful-stopping trials. Averages of RTs, PFs, and RFDs for the target hand in these three trial types are

\footnotetext{
$\overline{2}$ We excluded correct trials on the basis of the following criteria: (a) RT $<50 \mathrm{~ms}$ or RT $>800 \mathrm{~ms}(0.66 \%$ of correct trials $)$; (b) $\mathrm{PF}>$ $1500 \mathrm{cN}(0.45 \%$ of correct trials); or (c) the rate of force development was not computable because the PF was the first point exceeding $100 \mathrm{cN}(0.67 \%$ of correct trials).

${ }^{3}$ Previous evidence suggests that two separate responses are emitted as a single unit (i.e., "grouped") if the interval between them is $50 \mathrm{~ms}$ or less (e.g., Miller \& Ulrich, 2008). The average interval of $14 \mathrm{~ms}$ observed here was significantly less than $50 \mathrm{~ms}, t(19)=-53.99, p<$ 0.001 .
}

summarized in Table 1. Average force-time profiles are shown in Fig. 1.

The RT from a given hand was faster for unsuccessfulstopping trials than for no-stopping trials ( 417 vs. $444 \mathrm{ms,}$ respectively; $F(1,19)=31.82, p<0.001)$. This result agrees with previous findings in the stop-signal literature (e.g., Logan \& Cowan, 1984), and it is consistent with the race model in which the fastest RTs in the primary-task distribution lead to failed stops.

By definition, a given hand's PF was smaller in successfulthan in unsuccessful-stopping trials [30 and $310 \mathrm{cN}$, respectively; $F(1,19)=69.62, p<0.001]$. In addition, $\mathrm{PF}$ was smaller in unsuccessful-stopping than in no-stopping trials $(376 \mathrm{cN}), F(1,19)=21.83, p<0.001$. The RFD was also smaller in unsuccessful-stopping trials than in no-stopping trials $[2.89$ versus $3.35 \mathrm{cN} / \mathrm{ms}$, respectively; $F(1,19)=34.03$, $p<0.001]$. This reduced PF and RFD observed with unsuccessful stopping indicates that some inhibition was involved even though stopping was unsuccessful.

\section{Stopping-interference effects}

SIEs on the nontarget hand were also examined by comparing successful-stopping, unsuccessful-stopping, and no-stopping trials, although now the stopping success reflected the action of the other (i.e., target) hand. Averages of RTs, PFs, and RFDs for the nontarget hand in these three trial types are also shown in Table 1, and average forcetime profiles are shown in Fig. 1.

RTs from the nontarget hand were significantly different across the three trial types, $F(2,38)=143.89, p<0.001$. Further comparisons indicated that the RT from the nontarget hand was significantly larger in successful-stopping than in no-stopping trials $F(1,19)=256.06, p<0.001$, or in unsuccessful-stopping trials, $F(1,19)=149.10, p<0.001$. Thus, these RTs replicate Aron and Verbruggen's (2008) SIE. In addition, RTs from the nontarget hand were faster in unsuccessful-stopping than in no-stopping trials, $F(1,19)=$ $12.1, p<0.01$. This pattern matches the target hand and presumably reflects the tight coupling of the two responses described earlier.

PFs from the nontarget hand were significantly different among the three trial types, $F(2,38)=24.17, p<0.001$. Further comparisons indicated that nontarget hand $\mathrm{PF}$ was significantly larger in successful-stopping than in nostopping trials, $\mathrm{F}(1,19)=25.42, p<0.001$, or in unsuccessfulstopping trials, $F(1,19)=25.41, p<0.001$. Thus, an SIE was present in PF (i.e., increased PF on the nontarget hand in successful-stopping trials). RFDs from the nontarget hand were also significantly different among trial types, $F(2,38)=$ $39.88, p<0.001$. Nontarget hand RFD was significantly larger in successful-stopping trials than in no-stopping trials, $F(1,19)=30.19, p<0.001$, or in unsuccessful-stopping 
Fig. 1 Force-time profiles for each responding finger as a function of trial type. Force-time profiles of each trial were aligned to the point at which $50 \mathrm{cN}$ was reached before averaging, and this time-point is labeled "time zero"
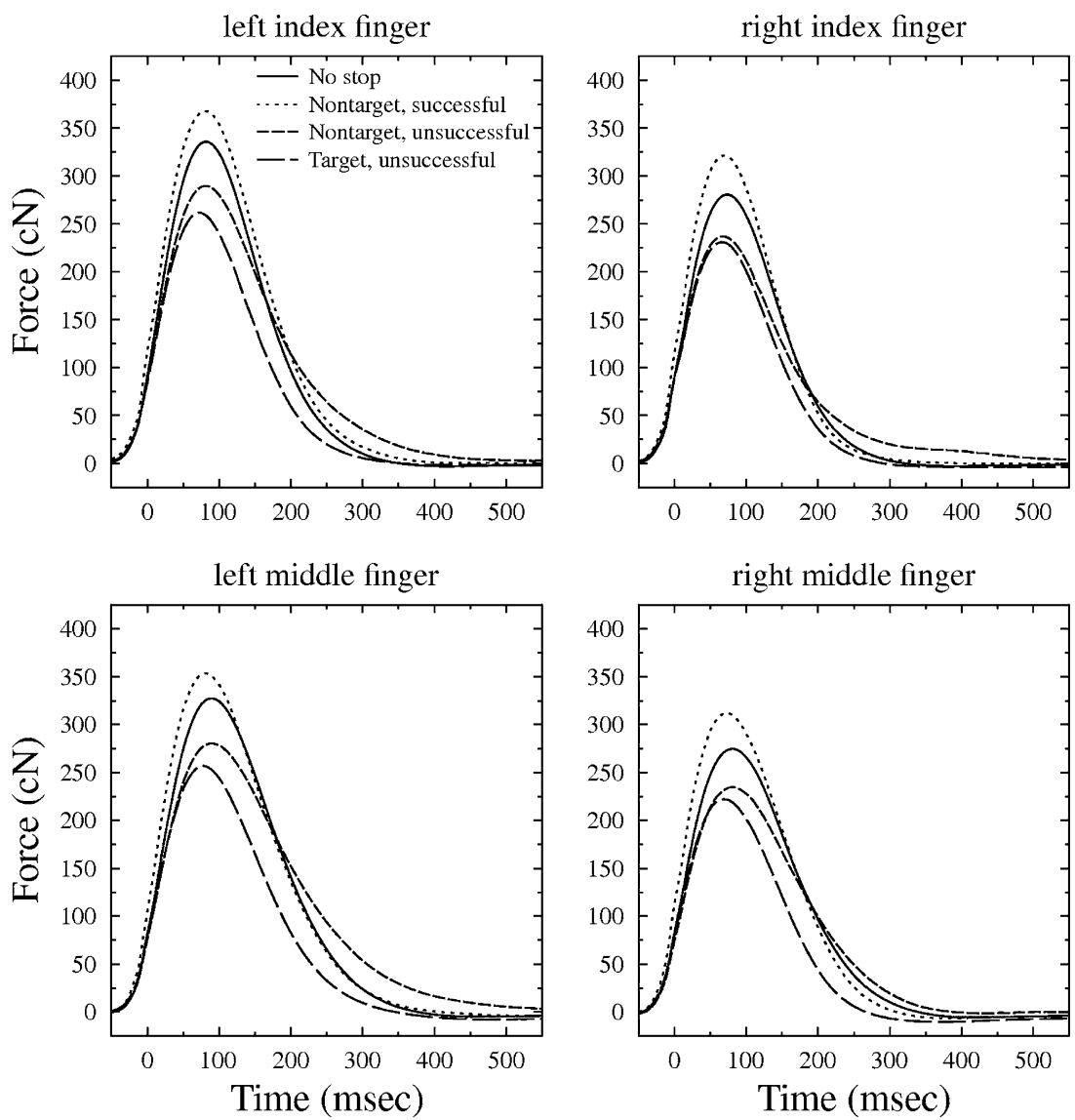

trials, $F(1,19)=48.45, p<0.001$. Thus, an SIE was also present in the rate of force development (i.e., increased nontarget hand RFD with successful stopping).

In addition, nontarget hand PFs were smaller in unsuccessful-stopping than in no-stopping trials, $F(1,19)=$ $8.8, p<0.01)$. Nontarget hand RFDs were also smaller in unsuccessful-stopping than in no-stopping trials, $F(1,19)=$
$30.9, p<0.01$.This pattern of nontarget hand results matches the target hand, consistent with the fact that the two hand response tendencies were concurrently processed. More importantly, this comparison between unsuccessful-stopping and no-stopping trials demonstrates that the nontarget hand response was affected, even when stopping for the target hand was unsuccessful.
Table 1 Means and standard deviations of reaction times (RT) in milliseconds (ms), mean peak force (PF) in centi-Newtons $(\mathrm{cN})$, and mean rate of force development (RFD) in the period from the response to the PF in centi-Newtons per millisecond $(\mathrm{cN} / \mathrm{ms})$ as a function of experimental condition

\begin{tabular}{|c|c|c|c|c|c|c|c|c|c|c|c|c|}
\hline \multirow{4}{*}{ Condition } & \multicolumn{12}{|c|}{ Dependent Measure and Hand } \\
\hline & \multicolumn{4}{|l|}{$\mathrm{RT}$} & \multicolumn{4}{|l|}{$\mathrm{PF}$} & \multicolumn{4}{|l|}{ RFD } \\
\hline & \multicolumn{2}{|c|}{ Nontarget Hand } & \multicolumn{2}{|c|}{ Target Hand } & \multicolumn{2}{|c|}{ Nontarget Hand } & \multicolumn{2}{|c|}{ Target Hand } & \multicolumn{2}{|c|}{ Nontarget Hand } & \multicolumn{2}{|c|}{ Target Hand } \\
\hline & $M$ & SD & $M$ & SD & $M$ & $S D$ & $M$ & $S D$ & $M$ & $S D$ & $M$ & $S D$ \\
\hline $\begin{array}{l}\text { Stop signal—stopped } \\
\text { (successful stopping) }\end{array}$ & 549 & 61 & - & & 427 & 251 & 30 & 13 & 3.88 & 2.1 & - & \\
\hline $\begin{array}{l}\text { Stop signal-not stopped } \\
\text { (unsuccessful stopping) }\end{array}$ & 422 & 41 & 417 & 36 & 356 & 194 & 310 & 153 & 3.08 & 1.7 & 2.89 & 1.6 \\
\hline $\begin{array}{l}\text { No stop signal } \\
\text { (no stopping required) }\end{array}$ & 444 & 37 & - & 376 & 220 & - & & 3.35 & 1.8 & - & & \\
\hline
\end{tabular}

Standard deviations are based on between-participant variation in the averages for each condition 
Baseline force differences: both hands

Several analyses were carried out to examine the possibility, suggested by an anonymous reviewer, that some observed PF differences might result from differences in baseline levels of force in the 200-ms preceding primary-task stimulus onset. Most importantly, the patterns of all PF and RFD comparisons are exactly the same regardless of whether a correction is applied for the baseline force level. Three interesting effects did emerge from the examination of baseline forces, however. First, the baseline force levels of the two hands were highly correlated across trials $(p<0.01$ for every participant, mean $r$ of 0.6), suggesting that the two responses may be prepared or preactivated as a single unit. Second, baseline force did not predict RT, suggesting that such preparation is not very important for response. Third, baseline force did predict stopping success. For the nontarget hand, the mean forces during baseline were 19.1, 19.6, and $19.8 \mathrm{cN}$ in successfulstopping, no-stopping, and unsuccessful-stopping trials, respectively, and these differences were significant, $F(2$, $38)=3.48, p<0.05$. Similarly, the target-hand baseline forces were $18.8,19.6$, and $20.0 \mathrm{cN}$ in successful-stopping, no-stopping, and unsuccessful-stopping trials, respectively, $F$ $(2,38)=9.07, p<0.01$. Together, the results from both hands indicate that stopping success was determined partly by the baseline level of motor activation, with lower force predicting more successful stopping.

\section{Discussion}

Peripheral inhibition in selective inhibition

In the present experiment, like in previous studies (Aron \& Verbruggen, 2008; Coxon et al., 2007), we observed that the nontarget-hand RT was longer when target response stopping was required and was successful than when no stopping was required, indicating that some process required for selective inhibition of target responses also slowed nontarget responses. We also found that nontarget response force was larger - and developed faster-when the target hand was stopped than when no stopping was required, indicating that selective inhibition of the target response affects nontarget responding at the motor level. Given previous evidence that response force is determined at or after M1 (e.g., Ashe, 1997), these force differences demonstrate that the processes responsible for the SIE have consequences for relatively peripheral motor processing. The present findings thus extend previous EMG evidence for that conclusion (Coxon et al., 2007) to a more standard selective stopping paradigm, and measures of motor activity that can be measured on single trials and are thus immune to contaminating effects of latency jitter.
Global inhibition and response overshoot

Because successful stopping of the target response also produces changes in the characteristics of the nontarget response, it appears that selective stopping involves a global inhibitory process rather than a completely local (i.e., response-specific) one (Aron \& Verbruggen, 2008). On the basis of the idea of a global inhibitory process, though, it is perhaps somewhat surprising that the nontarget hand force was increased rather than decreased by the processes responsible for the selective response inhibition. One might expect the result of a global inhibitory process to be a reduction in the forcefulness of all responses that are emitted. Obviously, however, this expectation is not correct.

A plausible account for the increased nontarget hand response force is that it emerges from the global component of the selective response inhibition process. When a stop signal appears in a selective-stopping task without a cue as to which response might be stopped, Aron and Verbruggen (2008) suggested that participants initially suppress all current response tendencies until they can determine which response still needs to be executed. Then, the nontarget hand would need to be reactivated so that it could be executed, and the increased nontarget-hand response force would emerge if the reactivation process were to overshoot the levels of activation and force typically produced in the absence of any stop signal. Although this overshoot model may at first appear rather ad hoc, it is essentially the same as the overshoot model of Mattes, Ulrich, and Miller (1997). Those authors found that both RT and response force increased as response probability decreased, suggesting that more weakly preactivated responses receive especially large activation boosts so that they are executed more forcefully.

Prestimulus motor activation affects success of stopping

Analyses of force levels generated during the baseline period prior to primary-task stimulus onset indicate that these forces are lower in trials for which stopping is successful than in trials for which it is not, and this difference is present for both the target and the nontarget hands. As was mentioned earlier, these differences indicate that the success or failure of stopping is determined partly by the participants' level of motor activation, as measured by key press force, at the moment of stimulus onset. It is known that participants make strategic pretrial adjustments to balance the conflicting goals of fast responding and high stopping probability (e.g., Verbruggen \& Logan, 2009), and these baseline period force differences suggest that some of these adjustments take place at a motor level. Motor-level adjustments of a specific response have also been demonstrated when foreknowledge indicates that that response might need to be stopped (Claffey, Sheldon, Stinear, Verbruggen, \& Aron, 2010). 
In conclusion, the present results clearly indicate that selective stopping of one response has motor-level consequences for the production of an unstopped response. The latter's force develops more rapidly in the duration from the response to the PF and ultimately attains a greater overall level. It follows, then, that even selective stopping of individual responses involves a type of nonselective global inhibition, and that this inhibition has consequences for the motor stage of primary-task processing for unstopped responses.

\section{References}

Aron, A. R., \& Verbruggen, F. (2008). Stop the presses: dissociating a selective from a global mechanism for stopping. Psychological Science, 19, 1146-1153.

Ashe, J. (1997). Force and the motor cortex. Behavioural Brain Research, 86, 1-15.

Band, G. P. H., \& Van Boxtel, G. J. M. (1999). Inhibitory motor control in stop paradigms: review and reinterpretation of neural mechanisms. Acta Psychologica, 101, 179-211.

Callaway, E., Halliday, R., Naylor, H., \& Thouvenin, D. (1984). The latency of the average is not the average of the latencies. Psychophysiology, 21, 571.

Claffey, M. P., Sheldon, S., Stinear, C. M., Verbruggen, F., \& Aron, A. R. (2010). Having a goal to stop action is associated with advance control of specific motor representations. Neuropsychologia, 48, 541-548.
Coxon, J. P., Stinear, C. M., \& Byblow, W. D. (2007). Selective inhibition of movement. Journal of Neurophysiology, 97, 2480-2489.

De Jong, R., Coles, M. G. H., \& Logan, G. D. (1995). Strategies and mechanisms in non-selective and selective inhibitory motor control. Journal of Experimental Psychology: Human Perception and Performance, 21, 498-511.

De Jong, R., Coles, M. G. H., Logan, G. D., \& Gratton, G. (1990). In search of the point of no return: the control of response processes. Journal of Experimental Psychology: Human Perception and Performance, 16, 164-182.

Giray, M., \& Ulrich, R. (1993). Motor coactivation revealed by response force in divided and focused attention. Journal of Experimental Psychology: Human Perception and Performance, 19, 1278-1291.

Logan, G. D., \& Cowan, W. B. (1984). On the ability to inhibit thought and action: A theory of an act of control. Psychological Review, 91, 295-327.

Logan, G. D., Schachar, R. J., \& Tannock, R. (1997). Impulsivity and inhibitory control. Psychological Science, 8, 60-64.

Mattes, S., Ulrich, R., \& Miller, J. (1997). Effects of response probability on response force in simple RT. Quarterly Journal of Experimental Psychology, 50A, 405-420.

Miller, J., \& Ulrich, R. (2008). Bimanual response grouping in dualtask paradigms. Quarterly Journal of Experimental Psychology, 61, 999-1019.

Pashler, H. E. (1994). Dual-task interference in simple tasks: data and theory. Psychological Bulletin, 116, 220-244.

Verbruggen, F., \& Logan, G. D. (2008). Response inhibition in the stop-signal paradigm. Trends in Cognitive Sciences, 12, 418-424.

Verbruggen, F., \& Logan, G. D. (2009). Proactive adjustments of response strategies in the stop-signal paradigm. Journal of Experimental Psychology: Human Perception \& Performance, $35,835-854$. 